\title{
Differentiating Neuroblastoma
}

National Cancer Institute

\section{Source}

National Cancer Institute. Differentiating Neuroblastoma. NCI Thesaurus. Code C42048.

A neuroblastoma in which the differentiating neuroblasts constitute more than fivepercent of the tumor cells. 